\title{
Influence of tert-amine groups on the solubility of polymers in $\mathrm{CO}_{2}$
}

\author{
Sevgi Kilic $^{\text {a,* }}$, Yang Wang ${ }^{\text {b }}$, J. Karl Johnson ${ }^{\text {b,c }}$, Eric J. Beckman ${ }^{\text {b }}$, Robert M. Enick ${ }^{\text {b,c }}$ \\ a Department of Chemical Engineering, Izmir Institute of Technology, Urla 35430, Izmir, Turkey \\ ${ }^{\mathrm{b}}$ Department of Chemical Engineering, University of Pittsburgh, Pittsburgh, PA 15261, USA \\ ${ }^{\mathrm{c}}$ National Energy Technology Laboratory, Pittsburgh, PA 15236, USA
}

\section{A R T I C L E I N F O}

\section{Article history:}

Received 19 January 2009

Received in revised form

4 March 2009

Accepted 7 March 2009

Available online 19 March 2009

\section{Keywords:}

Tertiary amine

$\mathrm{CO}_{2}$-solubility

$A b$ initio modeling

\begin{abstract}
A B S T R A C T
There is a need to develop new, non-fluorous polymers that are highly soluble in $\mathrm{CO}_{2}$. Experimental evidence indicates that tertiary amine and pyridine groups may exhibit favorable Lewis acid-Lewis base type interactions with $\mathrm{CO}_{2}$. It is therefore reasonable to assume that incorporation of tertiary amines into the side chain or backbone of non-fluorous polymers may impart a degree of $\mathrm{CO}_{2}$-solubility to the polymer. We present experimental results for eight different tert-amine-containing polymers. Of these polymers, only propyl dimethylamine-functionalized poly(dimethylsiloxane) is soluble in $\mathrm{CO}_{2}$ at temperatures and pressures accessible in our experiments, but even this polymer is less soluble than non-functionalized poly(dimethylsiloxane) at the same chain length. We have performed $a b$ initio calculations on tertiary amine-containing moieties representative of some of the polymers examined experimentally. Our calculations confirm that amine- $\mathrm{CO}_{2}$ interactions are indeed energetically favorable. However, we also find that the moiety self-interactions are typically more favorable than the $\mathrm{CO}_{2}$-moiety interactions. This indicates that the lack of solubility of amine-containing polymers in $\mathrm{CO}_{2}$ is a direct result of strong polymer-polymer interactions.
\end{abstract}

(c) 2009 Elsevier Ltd. All rights reserved.

\section{Introduction}

Polyfluoroacrylates and polyfluorophosphazines are among the most $\mathrm{CO}_{2}$-soluble polymers that have been identified to date [1]. Perfluoropolyethers are also quite miscible with $\mathrm{CO}_{2}$, although the cloud point pressures associated with these polymers increase dramatically as molecular weight increases above 10,000 [2]. Poly(dimethylsiloxane) (PDMS) has the highest solubility of any known non-fluorinated polymers [3-6]. Spectroscopic [7-10] and theoretical [11-13] investigations have provided insight related to the favorable thermodynamic interactions between dense $\mathrm{CO}_{2}$ and the fluorinated and siloxane-based polymers. There is a general consensus that the ability of these polymers to dissolve in $\mathrm{CO}_{2}$ is related to their low glass transition temperature, low cohesive energy density [14,15], and Lewis acid-Lewis base interactions between the polymers and $\mathrm{CO}_{2}[3,16]$.

There has been recent interest in identifying hydrocarbon-based polymers that also exhibit solubility in $\mathrm{CO}_{2}$ comparable to that of the fluorinated and siloxane-based polymers. Hydrocarbon-based polymers would likely have the advantages of being more environmentally benign and less expensive. Although no polymers

\footnotetext{
* Corresponding author.

E-mail addresses: sevgikilic@iyte.edu.tr (S. Kilic), rme@pitt.edu (R.M. Enick).
}

composed solely of carbon and hydrogen were found to be remarkably $\mathrm{CO}_{2}$-soluble, theoretical and experimental studies led to a handful of $\mathrm{CO}_{2}$-soluble oxygenated hydrocarbon polymers being identified [14]. Enhanced solubility of these polymers in $\mathrm{CO}_{2}$ was attributed to a specific interaction between the carbon atom of $\mathrm{CO}_{2}$ and the lone pairs on the oxygen of a carbonyl group, in which $\mathrm{CO}_{2}$ acts as a Lewis acid and the carbonyl group as a Lewis base [17]. This, in turn, led to studies [18-20] aimed at the inclusion of a carbonyl or acetate group in polymers to promote solubility in $\mathrm{CO}_{2}$ [21-26]. Subsequently, Raveendran and Wallen's ab initio calculations indicated that a second interaction, a cooperative interaction between oxygen atoms of $\mathrm{CO}_{2}$ and hydrogen atoms attached directly to the carbonyl carbon or the $\alpha$-carbon atom [27], was responsible for the superior $\mathrm{CO}_{2}$-philicity of carbonyl-based groups. Our group employed $a b$ initio calculations to reveal that the carbon of $\mathrm{CO}_{2}$ can interact to a comparable degree with the etherlike ester oxygen in the acetate group [6,14], confirming earlier reports that interactions of $\mathrm{CO}_{2}$ with this oxygen could occur $[28,29]$. The occurrence of multiple binding sites in an amorphous polymer with a low glass transition temperature is thought to be responsible for poly(vinyl acetate) displaying a greater degree of solubility in $\mathrm{CO}_{2}$ than any other high molecular weight oxygenated hydrocarbon [26]. We recently designed novel oligomers that dissolved in $\mathrm{CO}_{2}$, including polyvinyl methoxymethylether and poly(3-acetoxyoxetane) [30] and also demonstrated that 
poly(1-O-(vinyloxy)ethyl-2,3,4,6-tetra-O-acetyl- $\beta$-D-glucopyranoside) (a polymer with pendant sugar acetate groups) and amorphous polylactic acid are $\mathrm{CO}_{2}$-soluble over a wide range of molecular weight [31]. Nevertheless, poly(vinyl acetate) (PVAc) remains the most $\mathrm{CO}_{2}$-soluble polymer composed solely of carbon, hydrogen and oxygen that has yet been identified. Unfortunately, the pressure required to dissolve $\sim 5 \mathrm{wt} \%$ of $\mathrm{PVAc}$ in $\mathrm{CO}_{2}$ at $298 \mathrm{~K}$ is on the order of $50-70 \mathrm{MPa}$ [26], which is substantially higher than the pressure required to dissolve perfluoroacrylates or poly(dimethylsiloxane) (PDMS).

In an attempt to design other non-fluorous, non-silicone polymers that were soluble in $\mathrm{CO}_{2}$, we considered moieties composed of atoms other than $\mathrm{C}, \mathrm{H}, \mathrm{O}, \mathrm{N}, \mathrm{S}$ and $\mathrm{P}$ while retaining the fundamental strategy of exploiting $\mathrm{CO}_{2}$ behavior as a Lewis acid in the presence of a Lewis base $[18,19,20,32]$. While others have shown that trialkyl phosphate, trialkyl phosphite [33] and sulfonyls [27] may provide favorable interaction sites with $\mathrm{CO}_{2}$, we focused our efforts on aminated polymers. We note in passing that despite the $a b$ initio calculations by Raveendran and Wallen showing that the binding energy between $\mathrm{CO}_{2}$ and the $\mathrm{S}=\mathrm{O}$ sulfonyl group is much higher than that between $\mathrm{CO}_{2}$ and the carbonyl group, we found that sulfonyl functionalized poly(propylene glycol) exhibits much poorer miscibility with $\mathrm{CO}_{2}$ than the acetate-functionalized analogue [34].

Meredith et al. measured equilibrium constants by FT-IR spectroscopy for electron donor-acceptor interactions of $\mathrm{CO}_{2}$ with three Lewis bases: triethylamine, pyridine, and tributyl phosphate [35]. Their FT-IR spectroscopy results indicated that tributyl phosphate exhibits the strongest interactions with $\mathrm{CO}_{2}$-followed by pyridine and triethylamine. The authors stated that their density functional theory calculations, carried out to compare the relative stabilities and geometries of the $\mathrm{CO}_{2}$-triethylamine and $\mathrm{CO}_{2}$-pyridine complexes, also confirmed that $\mathrm{CO}_{2}$ interacts more strongly with pyridine than with triethylamine. They concluded that steric repulsion of the ethyl groups in triethylamine causes the binding energy of the $\mathrm{CO}_{2}$-triethylamine complex to be weaker than that of the $\mathrm{CO}_{2}$-pyridine complex, whereas the planar structure of pyridine leads to less steric hindrance, allowing the nitrogen atom to be closer to the carbon of $\mathrm{CO}_{2}$. The density functional theory formalism used by Meredith et al. to compute the $\mathrm{CO}_{2}$-amine interactions [35] lacks an accurate description of electron correlation effects that give rise to van der Waals interactions [36], which are expected to dominate the $\mathrm{CO}_{2}$-amine interaction; therefore the binding energies and perhaps even the geometries they report may be substantially in error. Furthermore, contrary to the results by Meredith et al., our $a b$ initio calculations, reported in this paper, indicate that interactions between $\mathrm{CO}_{2}$ and tert-amines are highly favorable-even stronger than $\mathrm{CO}_{2}$-carbonyl interactions. Therefore, one might expect that the tert-amine moiety incorporated into a polymer would have favorable Lewis acid-Lewis base type interactions with $\mathrm{CO}_{2}$, leading to miscibility of the tert-amine functionalized polymers with $\mathrm{CO}_{2}$.

Another indication that the amine group might enhance the solubility of a polymer in $\mathrm{CO}_{2}$ was associated with the electron donating capacity (donor number) of the compounds [37], which is a qualitative measure of Lewis basicity. Kachi et al. observed a linear relationship between electron donating capacity (donor number) of a molecule and the red-shift of the Raman bands of supercritical $\mathrm{CO}_{2}$, and suggested that the extent of the shift of the $\mathrm{CO}_{2}$ band can be used as a measure of $\mathrm{CO}_{2}$-philicity of solutes in supercritical $\mathrm{CO}_{2}$ [38]. According to the tabulated data, triethylamine has a donor number of $\mathrm{DN}=61$ (units of $\mathrm{kcal} / \mathrm{mol}$ ), which is very large compared to acetone $(\mathrm{DN}=17)$, diethyl ether $(\mathrm{DN}=19.2)$, ethyl acetate $(\mathrm{DN}=17.1)$, methyl acetate $(\mathrm{DN}=16.5)$, pyridine $(\mathrm{DN}=33.1)$, and tributyl phosphate $(\mathrm{DN}=23.7)[37,39]$. It is important to note that unlike primary and secondary amines, tertamines do not form carbamate salts with $\mathrm{CO}_{2}$ in the absence of water [40]. While such reactions may be desirable for the formation of surfactants upon reaction with $\mathrm{CO}_{2}$, our objective is the design of a polymer that dissolves in $\mathrm{CO}_{2}$ without reacting with the $\mathrm{CO}_{2}$. Therefore, we evaluated the $\mathrm{CO}_{2}$-solubility of several (polyethyleneimines) composed of $\mathrm{C}, \mathrm{H}, \mathrm{O}$ and $\mathrm{N}$. In addition to the requisite Lewis acid-Lewis base types of interactions likely to occur between poly(ethyleneimines) and $\mathrm{CO}_{2}$, poly(ethyleneimine)s are reported to exhibit weak self-interactions and relatively low glass transition temperature [41], and have a surface tension comparable to that of PDMS. For example, poly(hexanoyliminoethylene) and PDMS have surface tension values of 23 and $21 \mathrm{mN} / \mathrm{m}$ at $293 \mathrm{~K}$ respectively [41], and glass transition temperatures of $283 \mathrm{~K}$ and $150 \mathrm{~K}$, respectively, while fluoroacrylates have a glass transition temperature of $263 \mathrm{~K}$ and surface tension of $10 \mathrm{mN} / \mathrm{m}$ [41].

In summary, there are several indications that polymers containing tert-amines may show solubility in $\mathrm{CO}_{2}$. In order to test this hypothesis, we measured the $\mathrm{CO}_{2}$-solubility of seven hydrocarbonbased polymers possessing tert-amine groups either in the backbone or in the pendant chain. The hydrocarbon-based polymers we tested are listed in Table 1 . We also carried out complementary quantum mechanical calculations that are used to explore the atomic-level interactions in model polymer $/ \mathrm{CO}_{2}$ systems. We note that quantum chemical calculations alone cannot provide a complete description of the $\mathrm{CO}_{2}$-polymer system, but in this case it successfully explains our results where our design strategy fails.

\section{Experimental procedure}

The following section provides a description of the source or synthesis of the polymers investigated in this work.

\subsection{Materials}

Linear poly(ethyleneimine) hydrochloric salt $(\mathrm{Mn} \cong 2000)$ was a gift from Polymer Chemistry Innovations, Inc. N,N-dimethylacryl amide, 2,2,6,6-tetramethyl-1-piperidinyloxy (TEMPO, 99\%), anhydrous toluene, 2,2'-azobis(isobutyronitrile) (AIBN, 98\%) anhydrous $1 \mathrm{M} \mathrm{BH}_{3}$. THF (tetrahydrofuran) complex and methyl acrylate were obtained from Aldrich. N,N-dimethylacrylamide was purified by distillation under reduced pressure, and AIBN was purified by crystallization from ethanol prior to use.

Poly(2-ethyl-2-oxazoline) (PEOX) with a number average molecular weight of 5000 (50 repeat units) was purchased from Scientific Polymer Products, Inc. Poly(4-vinyl pyridine) (P4VP) $(\mathrm{Mn}=3000, \mathrm{PDI}=1.50)$, poly (2-vinyl pyridine $)(\mathrm{P} 2 \mathrm{VP})(\mathrm{Mn}=3000$, $\mathrm{PDI}=1.12)$, and poly $(\mathrm{N}-\mathrm{vinyl}$ imidazole $)(\mathrm{PVIZ}) \quad(\mathrm{Mn}=9500$, $\mathrm{PDI}=3.00)$ were purchased from Polymer Source, Inc. All materials were used as received.

\subsection{Synthesis of poly(N,N-dimethylacrylamide) PDMAA}

N,N-dimethylacrylamide was polymerized following a procedure given by $\mathrm{Li}$ and Brittain [42]. $0.50 \mathrm{~g}$ (3.04 mmol) AIBN and $0.47 \mathrm{~g}$ (3.01 mmol) TEMPO, such that $[$ AIBN]/[TEMPO] $=1$, were charged into a $250 \mathrm{ml}$ round-bottomed, three-neck flask equipped with a condenser and argon feed. $25.0 \mathrm{~g}(0.25 \mathrm{~mol}) \mathrm{N}, \mathrm{N}$-dimethylacrylamide and $100 \mathrm{ml}$ anhydrous toluene were then added to the flask. The flask was then placed in an oil bath at $371 \mathrm{~K}$. Initially, the solution exhibited the orange color of TEMPO, but the color disappeared in less than $30 \mathrm{~min}$. After $14 \mathrm{~h}$ of polymerization, the product was precipitated into hexane. The polymer was re-dissolved in toluene and re-precipitated into hexane twice, followed by vacuum drying overnight. White, hygroscopic, polymer 
Table 1

Structure of nitrogen-containing hydrocarbon-based polymers.

\begin{tabular}{|c|c|}
\hline Name of the polymer & Structure \\
\hline \multicolumn{2}{|l|}{ Poly(propylethyleneimine) (PPEI) } \\
\hline \multicolumn{2}{|c|}{ Poly(propylmethylacrylate ethyleneimine) (PPMAEI) } \\
\hline \multicolumn{2}{|c|}{ Poly(2-ethyl-2-oxazoline) (PEOX); precursor of PPEI } \\
\hline \multicolumn{2}{|c|}{ Poly(N,N-dimethylacrylamide) (PDMAA) } \\
\hline \multicolumn{2}{|l|}{ Poly(2-vinyl pyridine) (P2VP) } \\
\hline \multicolumn{2}{|l|}{ Poly(4-vinyl pyridine) (P4VP) } \\
\hline \multicolumn{2}{|l|}{ Poly(N-vinyl imidazole) (PVIZ) } \\
\hline
\end{tabular}

powder was recovered at $99 \%$ yield. The molecular weight of poly(N,N-dimethylacrylamide) was determined via gel permeation chromatography using toluene as eluent $(\mathrm{Mn}=1298, \mathrm{Mw}=1672$, $\mathrm{PI}=1.29) .{ }^{1} \mathrm{H}$ NMR $\left(300 \mathrm{MHz}, \mathrm{C}_{6} \mathrm{D}_{6}\right): \delta 2.7-2.9$ (broad, $1 \mathrm{H},-\mathrm{CH}-\mathrm{CO}-$ $\left.\mathrm{N}-\left(\mathrm{CH}_{3}\right)_{2}\right), \delta$ 2.9-3.0 (broad, $\left.6 \mathrm{H},-\mathrm{N}-\left(\mathrm{CH}_{3}\right)_{2}\right), \delta$ 1.5-1.9 (broad, $2 \mathrm{H}$, $\left.-\mathrm{CH}_{2}-\mathrm{CH}-\mathrm{CO}\right)$.

\subsection{Synthesis of poly(propylethyleneimine) PPEI}

Poly(propylethyleneimine) was synthesized via the reduction of poly(2-ethyl-2-oxazoline) by borane (Scheme 1). The glassware

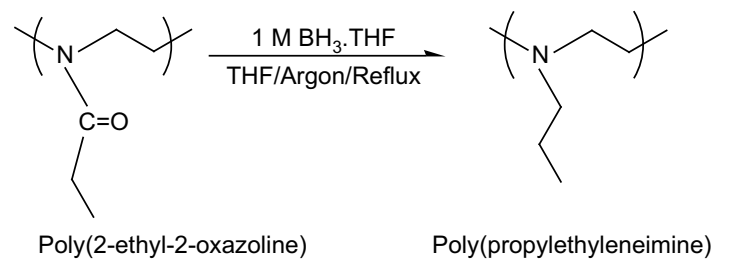

Scheme 1. Synthetic route for preparation of poly(propylethylenimine).

was oven-dried overnight and purged with ultra-high purity argon before use. $9.4 \mathrm{~g}$ of polymer was charged into a 500-ml three-neck, round-bottomed flask. The system was equipped with a magnetic stir-bar, a condenser, addition funnel, and an argon feed. $25 \mathrm{ml}$ of anhydrous THF were added to completely dissolve the polymer. After dissolution, $430 \mathrm{ml} 1 \mathrm{M} \mathrm{BH}_{3} \cdot \mathrm{THF}$ complex (4.2 equivalent) were added to the flask drop-wise over $180 \mathrm{~min}$. The solution temperature was raised to reflux, and the solution was stirred for 4 days. After cooling, the excess borane was eliminated by dropwise addition of methanol until hydrogen gas ceased evolving. The $\mathrm{THF} /$ methanol mixture was evaporated under reduced pressure, and the sample was dissolved in $144 \mathrm{ml}$ of methanol. An aqueous solution of $\mathrm{HCl}(6 \mathrm{~N}, 48 \mathrm{ml}, 3$ times excess) was added and to the sample and the solution heated to $338 \mathrm{~K}$ and stirred for $40 \mathrm{~h}$. Upon cooling the green solution, $\mathrm{NaOH}$ aqueous solution $(6 \mathrm{~N}, 50 \mathrm{ml})$ was added to neutralize the mixture. Methanol was removed with a rotary evaporator under reduced pressure, and water by azeotropic distillation with toluene. The salt was removed by filtration after re-dissolving the polymer in methanol. In the event that some salt remained dissolved in the residual water after azeotropic distillation with toluene, the methanol was removed, and the polymer was dissolved in chloroform. The solution was dried over $\mathrm{MgSO}_{4}$. Upon removal of chloroform, a viscous, brown polymer was obtained (47\% yield). Disappearance of the peak at $1647 \mathrm{~cm}^{-1}$ (corresponding to $\mathrm{C}=\mathrm{O}$ stretching in poly(2-ethyl-2-oxazoline)) was a sign of complete reduction of the amide. ${ }^{1} \mathrm{H}$ NMR $(300 \mathrm{MHz}$, $\left.\mathrm{CDCl}_{3}\right) \delta 0.90$ (broad, $3 \mathrm{H},\left(-\mathrm{N}-\mathrm{CH}_{2}-\mathrm{CH}_{2}-\mathrm{CH}_{3}\right), \delta 1.5$ (broad, $4 \mathrm{H},-\mathrm{N}-$ $\mathrm{CH}_{2}-\mathrm{CH}_{2}-\mathrm{CH}_{3}$ ), $\delta 2.3-2.5$ (broad, $4 \mathrm{H}, \mathrm{N}-\mathrm{CH}_{2}$ ).

\subsection{Synthesis of poly(propylmethylacrylate ethyleneimine) PPMAEI}

Poly(propylmethylacrylate ethyleneimine) was prepared via reaction of poly(ethyleneimine) with methyl acrylate (known as 'Michael addition' reaction) [43-45]. Prior to the reaction, poly(ethyleneimine) hydrochloric salt was first neutralized with aqueous $\mathrm{NaOH}$ solution. For this, $11.0 \mathrm{~g}$ poly(ethyleneimine) hydrochloric salt was allowed to dissolve in $60 \mathrm{ml}$ water, then $5 \mathrm{~g}$ $\mathrm{NaOH}$ dissolved in $20 \mathrm{ml}$ water was added slowly to the polymer solution until the $\mathrm{pH}$ of the solution was 8.0-8.5, as determined by $\mathrm{pH}$ paper. The solution was stirred overnight. Upon precipitation of the polymer into acetone (twice), a yellowish viscous, oily polymer precipitated at the bottom of the flask. In case some salt remained dissolved in the residual water, the polymer was dissolved in methanol and dried over $\mathrm{K}_{2} \mathrm{CO}_{3}$. Upon filtration, excess methanol was removed with a rotary evaporator as needed. The Michael addition reaction was then employed. The solution ( $\sim 75 \mathrm{ml}$ ) was transferred to a $250-\mathrm{ml} 3$-neck, round bottom flask equipped with a condenser, and $18.5 \mathrm{~g}(0.22 \mathrm{~mol})$ of methyl acrylate was then added. Initially, the solution was opaque and yellow in appearance, but after $72 \mathrm{~h}$ of stirring, the color turned to orange. After filtration, the solution was concentrated under vacuum to remove unreacted methyl acrylate and methanol. A very viscous, red-brownish polymer was obtained $(92 \%$ yield). ${ }^{1} \mathrm{H}$ NMR (300 MHz, $\left.\mathrm{CDCl}_{3}\right) \delta 1.5$ (broad, $2 \mathrm{H},-\mathrm{N}-\mathrm{CH}_{2}-\mathrm{CH}_{2}$ ), 
$\delta$ 2.3-2.5 (broad, $4 \mathrm{H},-\mathrm{N}-\mathrm{CH}_{2}$ ), $\delta$ 2.5-2.9 (broad, $2 \mathrm{H}, \mathrm{N}-\mathrm{CH}_{2}-\mathrm{CH}_{2}-$ $\mathrm{CO}), \delta 3.7\left(\mathrm{~s}, 3 \mathrm{H}, \mathrm{CO}-\mathrm{O}-\mathrm{CH}_{3}\right)$.

\subsection{Synthesis of functional siloxane copolymers}

In addition to the seven hydrocarbon-based polymers containing tert-amine groups discussed above, dimethylsiloxane polymer was functionalized via the addition of acetate- or tert-amine-containing groups in order to assess the influence of these two $\mathrm{CO}_{2-}$ philic groups on the cloud point pressure of a highly $\mathrm{CO}_{2}$-soluble siloxane polymer.

Propyl acetate (PA) and propyl dimethylamine (PDMA) functional siloxane copolymers were prepared according to the procedure described earlier [6].

\subsection{Phase behavior measurements}

Phase behavior measurements of the polymers were performed in the same way as described earlier [6]. Typical variability in the cloud point measurement is less than $\pm 0.7 \mathrm{MPa}$.

\section{Simulation methods}

We used Møller-Plesset second order perturbation theory (MP2) and the $6-31+\mathrm{G}(\mathrm{d})$ basis set to perform geometry optimization calculations, as implemented within the Gaussian 03 suite of programs [46]. We have found that the MP2 level of theory gives results in fairly good agreement with coupled-cluster with single, double, and perturbative triple excitations calculations on similar systems [6]. A larger basis set, aug-cc-pVDZ, was used for single point energy calculations on the $6-31+G(d)$ optimized structures. The counterpoise (CP) method [47] was used to correct for basis set superposition errors. We used the average of the $\mathrm{CP}$-corrected and uncorrected energies to approximate the energy at complete basis set limit. This approach is less computationally expensive than basis set extrapolation and has been shown to be accurate in our previous calculations [6]. Feller and Jordan have also used this method for approximating complete basis set energies [48].

It is not currently feasible to carry out extensive MP2 calculations on molecules containing more than about 10 heavy atoms. We have therefore used three moderately sized tertiary amine molecules to approximate the interactions of $\mathrm{CO}_{2}$ with poly(propylethyleneimine), poly(2-vinyl pyridine), and poly(4-vinyl pyridine). The representative molecules we have chosen are trimethylamine, 4-methyl pyridine, and 2-methyl pyridine; the structures of these molecules are shown in Fig. 1.

\section{Results and discussion}

Interactions of $\mathrm{CO}_{2}$ with the functional groups in Fig. 1 have been computed, along with the self-interactions for each of the three moieties. These calculations alone are not capable of predicting the solubilities of polymers in $\mathrm{CO}_{2}$ because self- and

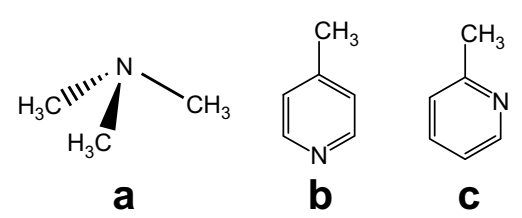

Fig. 1. Structures of three polymer moieties used in our $a b$ initio calculations. (a) Trimethylamine, a surrogate for poly(propylethyleneimine). (b) 4-Methyl pyridine, a surrogate for poly(4-vinyl pyridine). (c) 2-Methyl pyridine, a surrogate for poly(2vinyl pyridine). cross-interaction energies are only part of the picture. Entropic and cooperative effects must be considered within the framework of statistical mechanics. Thus, our quantum mechanical calculations can only serve as a guide to understanding solubility trends.

Only one binding mode has been identified for the trimethylamine $/ \mathrm{CO}_{2}$ complex (see Fig. 2). The calculated interaction energy is $-19 \mathrm{~kJ} / \mathrm{mol}$. To put this binding energy in context, we note that interaction of $\mathrm{CO}_{2}$ with the carbonyl oxygen of isopropyl acetate (IPA) is $-15.9 \mathrm{~kJ} / \mathrm{mol}[16]$ and that IPA is a surrogate for poly(vinyl acetate), a polymer that has been identified as having the highest $\mathrm{CO}_{2}$-solubility of any known oxygenated hydrocarbon polymer (a polymer containing only carbon, oxygen, and hydrogen) [16].

The results of the binding energy calculations for trimethylamine $/ \mathrm{CO}_{2}$ indicate that poly(propylethyleneimine) may be soluble in $\mathrm{CO}_{2}$. However, one must also take into account the polymerpolymer interactions, which we approximate by computing the dimer energies for trimethylamine. We have found two binding modes for the trimethylamine dimer, which we identify as modes A and B. These modes are illustrated in Fig. 3.

The two binding modes both show strong self-interactions of $-14.0 \mathrm{~kJ} / \mathrm{mol}$ and $-21.1 \mathrm{~kJ} / \mathrm{mol}$ for configuration $\mathrm{A}$ and $\mathrm{B}$, respectively. The interaction energy of binding mode $B$ is stronger than the interaction energy between trimethylamine and $\mathrm{CO}_{2}$. We also note that the existence of these two binding modes will sterically hinder the $\mathrm{CO}_{2}$-trimethylamine interaction. Note that a third trimethylamine molecule can be added symmetrically to the molecule on the right side in the binding mode $\mathrm{B}$ and this will completely block the $\mathrm{CO}_{2}$ binding site of the molecule on the left side. Hence, it is likely that the self-interactions among the amine side chains in poly(propylethyleneimine) will weaken their interactions with $\mathrm{CO}_{2}$ molecules, in turn leading to reduced $\mathrm{CO}_{2}-$ solubility.

We have optimized the pyridine $/ \mathrm{CO}_{2}$ systems and have identified a single minimum for each of the pyridine structures. The binding modes are shown in Fig. 4. The interaction energies are computed to be -19.1 and $-21.2 \mathrm{~kJ} / \mathrm{mol}$ for 4 -methyl pyridine $/ \mathrm{CO}_{2}$ and 2-methyl pyridine $/ \mathrm{CO}_{2}$, respectively. These energies are comparable to the interaction energy of trimethylamine $/ \mathrm{CO}_{2}$ complex and stronger than those of IPA/ $\mathrm{CO}_{2}$ system [14].

We have also investigated the self-interactions between two pyridine molecules. We have carried out calculations with dimers of the 4-methyl pyridine molecule. The dimer energies of the 2methyl pyridine molecule are expected to be very similar to those for 4-methyl pyridine and so are not calculated here. Two optimized binding structures have been found for 4-methyl pyridine self-interaction (see Fig. 5), which we identify as modes A and B. The two pyridine rings are parallel to each other for mode $A$, and are perpendicular to each other for mode $B$. The binding energy for
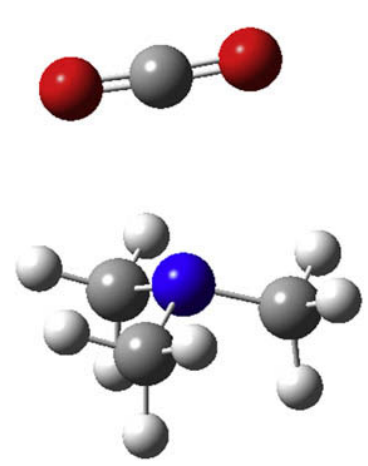

Fig. 2. Optimized binding structure for trimethylamine $/ \mathrm{CO}_{2}$ complex. Interaction energy $=-19 \mathrm{~kJ} / \mathrm{mol}$. 

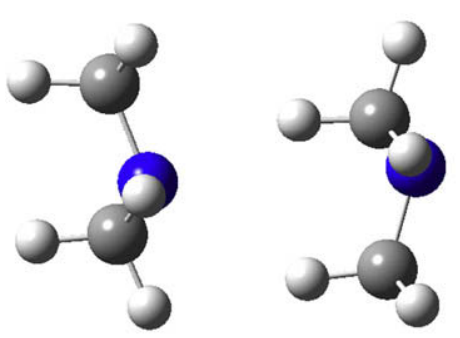

Mode A side view

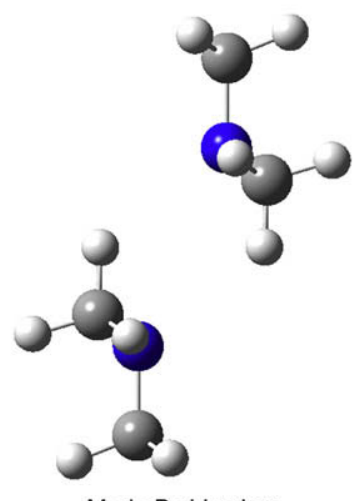

Mode B side view

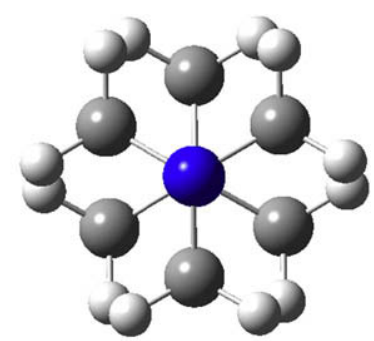

Mode $\mathrm{A}$ end on view

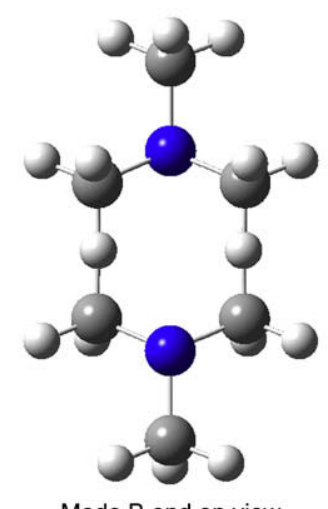

Mode $B$ end on view
Fig. 3. Side views and end on views of two different optimized binding structures, denoted $\mathrm{A}$ and $\mathrm{B}$, for the trimethylamine dimer. Binding mode $\mathrm{A}$ has an interaction energy of $-14.0 \mathrm{~kJ} / \mathrm{mol}$; Binding mode $B$ has an interaction energy of $-21.1 \mathrm{~kJ} / \mathrm{mol}$.

the parallel ring configuration was calculated to be $-42.8 \mathrm{~kJ} / \mathrm{mol}$. The interaction energy of the perpendicular ring configuration has a value of $-18.1 \mathrm{~kJ} / \mathrm{mol}$.

Note that the perpendicular configuration has a binding energy that is comparable to the pyridine $/ \mathrm{CO}_{2}$ interaction energies, whereas the parallel structure binding is about a factor of two more strongly bound than the pyridine $/ \mathrm{CO}_{2}$ dimers. This result suggests that the polymer/polymer interactions are dominant over the polymer $/ \mathrm{CO}_{2}$ interactions. Hence, we would expect that polymers with pyridine-like side groups would not exhibit high solubility in $\mathrm{CO}_{2}$, since the very strong polymer-polymer interactions would likely prohibit $\mathrm{CO}_{2}$ from solubilizing the polymer.
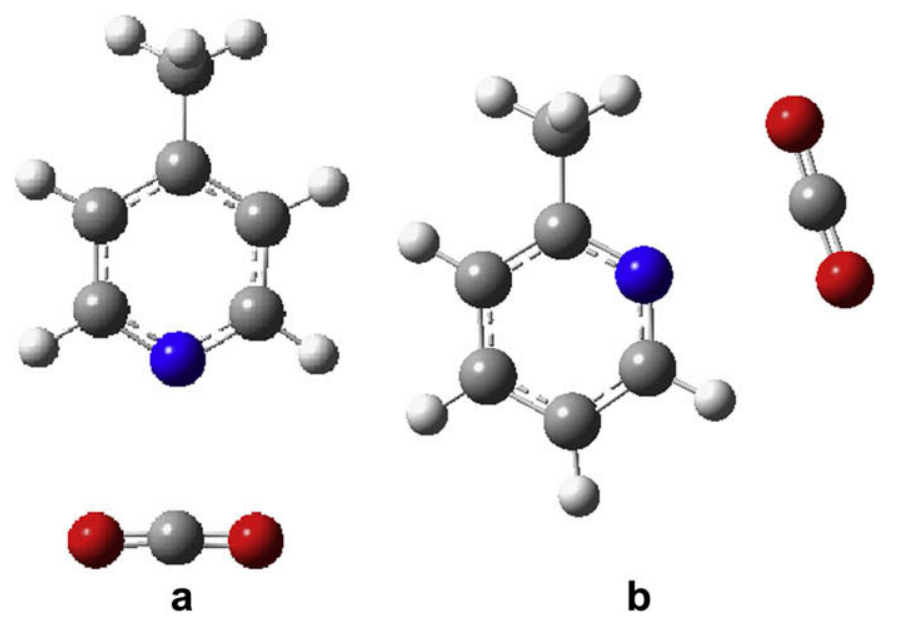

Fig. 4. Optimized binding structures for 4-methyl pyridine $/ \mathrm{CO}_{2}$ (a) and 2-methyl pyridine $/ \mathrm{CO}_{2}$ (b) systems. The interaction energies are -19.1 and $-21.2 \mathrm{~kJ} / \mathrm{mol}$, respectively.
A summary of our $a b$ initio calculation results for the interaction energies of the systems investigated is presented in Table 2.

We have evaluated $\mathrm{CO}_{2}$-solubility of seven hydrocarbon-based polymers containing nitrogen, either in the backbone or in the side chain, (see Table 1 for structures) as well as that of propyl dimethyamine (PDMA) and propyl acetate (PA) functionalized siloxane polymers (see Fig. 7 for structures). We evaluated their solubility in $\mathrm{CO}_{2}$ at conditions where $\mathrm{CO}_{2}$ is a dense liquid at $295 \mathrm{~K}$ and in some cases at supercritical conditions.

We have tested the solubility of the three ethyleneimine polymers: poly(propylethyleneimine) (PPEI), poly(propylmethylacrylate ethyleneimine) (PPMAEI) and poly(2-ethyl-2-oxazoline) (PEOX). The number of repeat units for all these three polymers is about the same ( $\sim 47-50$ repeat units). The structures of these polymers are shown in Table 1 . None of these polymers were soluble in $\mathrm{CO}_{2}$ at $295 \mathrm{~K}$ down to $1 \mathrm{wt} \%$ and pressures up to $45 \mathrm{MPa}$. The result for PPEI is consistent with our ab initio calculations summarized in Table 2, showing that trimethylamine self-interactions dominate over trimethylamine- $\mathrm{CO}_{2}$ cross interactions. Moreover, only one binding mode was found for the $\mathrm{CO}_{2}$ /trimethylamine system shown in Fig. 2. This implies that even if the site is not blocked by amine self-interactions, only a single $\mathrm{CO}_{2}$-amine bond per repeat unit is probably insufficient to induce solubility of the polymer. PPMAEI and PEOX have $\mathrm{CO}_{2}$-philic acrylate and carbonyl side groups, respectively. We had postulated that these groups would induce $\mathrm{CO}_{2}$-solubility of the polymers [14]. Unfortunately, even the addition of these groups failed to achieve any measurable solubility. The lack of solubility is not entirely unexpected for PPMAEI for two reasons. Firstly, an ethylene group separates the acrylate group from the backbone and we know that insertion of a methylene group between the backbone and the acetate group of poly(vinyl acetate), resulting in poly(allyl acetate), has a remarkable deleterious effect on miscibility of poly(allyl acetate) in $\mathrm{CO}_{2}$ [14]. Secondly, the acrylate group is known to be much less effective than an acetate group at imparting $\mathrm{CO}_{2}$-solubility from the comparison of poly(vinyl acetate) versus poly(methyl acrylate) [26]. For PEOX the lack of an oxygen atom between the backbone and the carbonyl group diminishes side chain rotational motion, and hence the free volume of the polymer, leading to less favorable interactions between $\mathrm{CO}_{2}$ and the polymer side chain [6].

In order to test whether steric hindrance plays a role in the $\mathrm{CO}_{2}$-solubility of tert-amine-containing polymers (the $\mathrm{N}$ is in the backbone of PPEI, PPMAEI, and PEOX), we measured the solubility of PDMAA in $\mathrm{CO}_{2}$. PDMAA was synthesized with a mole cular weight of 1298 (13 repeat units). PDMAA, unfortunately, was found to be immiscible with $\mathrm{CO}_{2}$ at pressures of $45 \mathrm{MPa}$ and concentrations as low as $0.7 \mathrm{wt} \%$ at $295 \mathrm{~K}$. The polymer was swollen to some degree by $\mathrm{CO}_{2}$. This may be an indication for the formation of a polymeric network structure as a result of selfinteractions between the polymer chains, with the $\mathrm{CO}_{2}$ molecules having been encapsulated within, possibly via carbonyl- $\mathrm{CO}_{2}$ and tert-amine- $\mathrm{CO}_{2}$ interactions. Increasing the temperature to $353 \mathrm{~K}$ failed to produce a single phase solution. The immiscibility of PDMAA can be attributed to the very high cohesive energy density of the polymer (surface tension: $\sim 52 \mathrm{mN} / \mathrm{m}$ at $293 \mathrm{~K}$ ) and very high $T_{g}(362 \mathrm{~K})$ [41], meaning that entropic and enthalpic factors favor immiscibility. Here again, Lewis acidLewis base interactions of $\mathrm{CO}_{2}$ with the carbonyl or the tertamine group were insufficient to overcome the self-interactions between polymer chains.

We hypothesized that the tert-amine group strengthens the Lewis basicity of the carbonyl group through its electron donor character, and thereby increases carbonyl $\mathrm{CO}_{2}$-philicity while weakening its own $\mathrm{CO}_{2}$-philicity. Indeed, our $a b$ initio calculations for the natural bond order charge distributions of N,N-dimethyl 

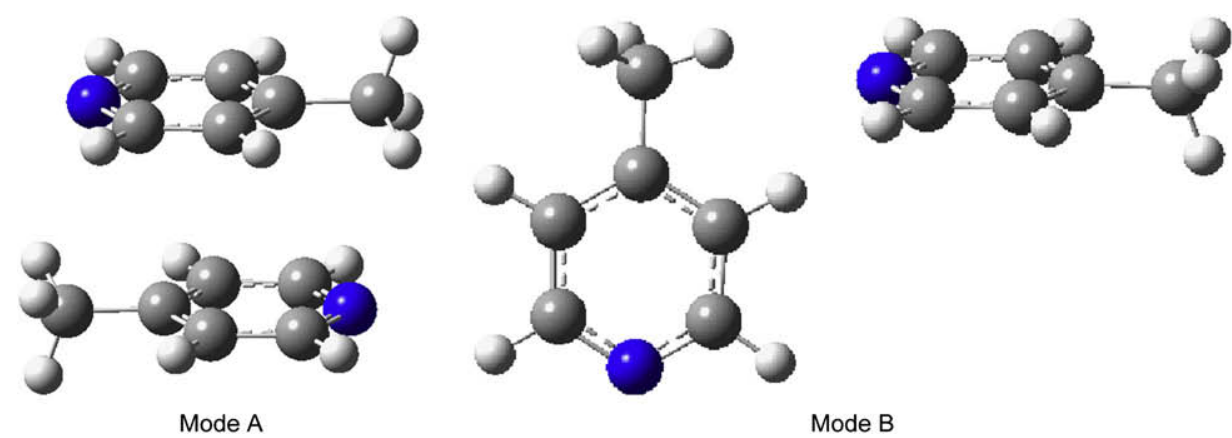

Mode B

Fig. 5. Optimized binding structures for 4-methyl pyridine dimer. For mode A the interaction energy is $-42.8 \mathrm{~kJ} / \mathrm{mol}$. For mode $\mathrm{B}$ the interaction energy is $-18.1 \mathrm{~kJ} / \mathrm{mol}$.

acetamide support this hypothesis. The nitrogen atom is less electron rich, having a charge of $-0.526 \mathrm{e}$, compared with -0.571 in trimethylamine. The oxygen atom in N,N-dimethyl acetamide is a stronger Lewis base $(-0.616)$ compared with the carbonyl oxygen $(-0.534)$ in methyl-isobutyl-ketone.

The polymers we have considered so far have all been tertiary amines. Meredith and coworkers[35] reported that interactions between $\mathrm{CO}_{2}$ and triethylamine are not as favorable as between $\mathrm{CO}_{2}$ and pyridine, because the ethyl groups in triethylamine sterically hinder the $\mathrm{CO}_{2}$ molecule from getting as close to the nitrogen atom in triethylamine as in pyridine [35]. This observation was based on gas-phase density functional theory (DFT) calculations. The DFT calculations utilized the B3LYP generalized gradient approximation [49]. They reported that the $\mathrm{C}$ atom in $\mathrm{CO}_{2}$ was $0.27 \AA$ A closer to the nitrogen in $\mathrm{CO}_{2} /$ pyridine than in $\mathrm{CO}_{2} /$ triethylamine. Moreover, they reported that the $\mathrm{CO}_{2}$ /pyridine complex was more strongly bound by $5.6 \mathrm{~kJ} / \mathrm{mol}$ than the $\mathrm{CO}_{2} /$ triethylamine complex [35]. However, the calculations performed by Meredith et al. may be in error because DFT is known not to be accurate for weakly bound systems because the electron correlation treatment in B3LYP has the wrong long-range behavior, and therefore cannot capture the correct van der Waals dispersion interactions [36]. Hence, for supramolecular systems in which the binding is dominated by dispersive interactions, both the geometries and binding energies may be in error. Our $a b$ initio calculations using the MP2 level of theory show that the interaction energy for $\mathrm{CO}_{2} /$ trimethylamine is about the same as that for $\mathrm{CO}_{2} / 4$-methyl pyridine, and only $2.2 \mathrm{~kJ} / \mathrm{mol}$ less than the interaction between $\mathrm{CO}_{2}$ and 2-methyl pyridine (see Table 2). We also have optimized the geometry and computed the binding energy for the $\mathrm{CO}_{2}$ /triethylamine system in order to compare with the DFT calculations of Meredith et al. [35]. We found that the carbon-nitrogen distance is only $0.012 \AA$ larger for the $\mathrm{CO}_{2}$-triethylamine complex (shown in Fig. 6) compared with the $\mathrm{CO}_{2}-4$-methyl pyridine complex.

In further contrast to the DFT calculations of Meredith et al., the $\mathrm{CO}_{2}$-triethylamine binding energy is $-23.6 \mathrm{~kJ} / \mathrm{mol}$ and is therefore slightly more favorable than that between $\mathrm{CO}_{2}$ and either 4-methyl pyridine or 2-methyl pyridine. The slightly more favorable binding for the $\mathrm{CO}_{2}$-triethylamine complex compared with the pyridines or the trimethylamine is likely due to van der Waals interactions between $\mathrm{CO}_{2}$ and the adjacent ethylene groups. In other words, the primary Lewis acid-Lewis base interactions are about the same for all of these molecules and hence there is no evidence of steric hindrance decreasing the binding between $\mathrm{CO}_{2}$ and triethylamine. The larger distance and weaker interaction energy Meredith et al. [35] observed is very likely an artifact of using DFT to compute the interaction energies. Our previous calculations indicate that B3LYP DFT can be even worse than Hartree-Fock theory (which lacks electron correlation) for computing binding energies between $\mathrm{CO}_{2}$ and small molecules [6].

Our $a b$ initio calculations for the $\mathrm{CO}_{2} /$ pyridine systems predict that self-interactions between the pyridine groups will dominate over the Lewis acid-Lewis base interactions between $\mathrm{CO}_{2}$ and the nitrogen and therefore polymers that contain pyridine-like groups should not show high solubility in $\mathrm{CO}_{2}$. In order to test this hypothesis we tested the solubilities of poly(2-vinyl pyridine) (P2VP), poly(4-vinyl pyridine) (P4VP) and poly(N-vinyl imidazole) (PVIZ) in $\mathrm{CO}_{2}$. None of these polymers were found to be miscible with $\mathrm{CO}_{2}$ at a temperature of $295 \mathrm{~K}$ and pressures up to $55 \mathrm{MPa}$ and at concentrations down to $0.7 \mathrm{wt} \%$. Elevated temperature ( $343 \mathrm{~K}$ ) did not result in a single phase solution. The surface tensions of P2VP and P4VP were reported as 45 and $71.5 \mathrm{mN} / \mathrm{m}$ at $293 \mathrm{~K}$, respectively [50]. Hence, the polymer-polymer interactions appear to be too strong for the polymer to be soluble in $\mathrm{CO}_{2}$, in agreement with our $a b$ initio calculations.

We have tested a total of seven polymers containing only the elements $\mathrm{C}, \mathrm{H}, \mathrm{O}$, and $\mathrm{N}$, with the nitrogen atoms forming tertiary amine groups either in the backbone or in the side chain. None of these polymers were found to be soluble in $\mathrm{CO}_{2}$. In order to establish whether tert-amine groups increase or decrease the cloud point pressure of polymers known to be highly $\mathrm{CO}_{2}$-soluble, we have functionalized the backbone of PDMS with PDMA (Fig. 7). We also synthesized propyl acetate (PA) functionalized PDMS to compare the $\mathrm{CO}_{2}$-solubility of acetate-functionalized siloxane

Table 2

Summary of interaction energies for amine $/ \mathrm{CO}_{2}$ systems and amine self-interactions.

\begin{tabular}{|c|c|c|}
\hline & System & Interaction energy $(\mathrm{kJ} / \mathrm{mol})$ \\
\hline \multirow[t]{4}{*}{ Trialkylamine } & trimethylamine $/ \mathrm{CO}_{2}$ & -19.0 \\
\hline & triethylamine $/ \mathrm{CO}_{2}$ & -23.6 \\
\hline & trimethylamine self-interactions mode A & -14.0 \\
\hline & trimethylamine self-interactions mode B & -21.1 \\
\hline \multirow[t]{4}{*}{ Cyclic amine } & 4-methyl pyridine $/ \mathrm{CO}_{2}$ & -19.1 \\
\hline & 2-methyl pyridine $/ \mathrm{CO}_{2}$ & -21.2 \\
\hline & 4-methyl pyridine self-interaction mode $\mathrm{A}$ & -42.8 \\
\hline & 4-methyl pyridine self-interaction mode B & -18.1 \\
\hline
\end{tabular}



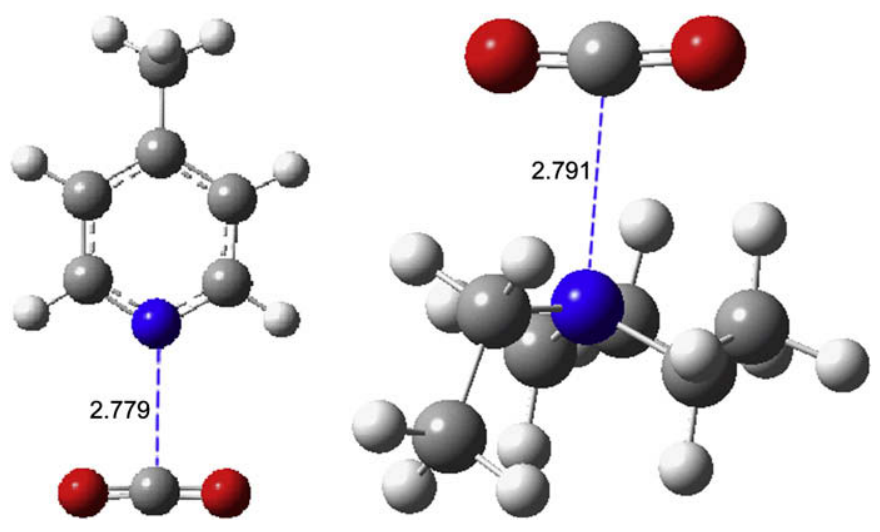

Fig. 6. The distances between the carbon and nitrogen atoms for $\mathrm{CO}_{2}-4$-methyl pyridine and $\mathrm{CO}_{2}$-triethylamine are 2.779 and $2.791 \AA$, respectively.

polymers with tert-amine-functionalized siloxane polymers, both having the same chain length, and the PA-functionalized PDMS having a slightly higher molecular weight at the same degree of substitution. We have previously shown that Lewis base functionalized (e.g., ether, ketone, carbonate, acetate, acrylate) siloxane polymers can be synthesized that have higher solubility in $\mathrm{CO}_{2}$ than PDMS at their optimum substitution [6]. Typically, we found that an optimum degree of functionalization exists as a result of a balance between factors suppressing miscibility (increased molecular weight, increased cohesive energy density) and factors promoting miscibility (Lewis acid-Lewis base interactions, free volume, chain flexibility, configuration entropy, etc.) [6]. We therefore synthesized siloxane polymers functionalized with either PDMA or PA side chains, having varying degrees of functionalization, as shown in Fig. 7. The siloxane polymers have a total of 25 repeat units with the degree of substitution $(z)$ varying from 1 to 25 . Thus, we investigated the effect of varying amounts of PDMA and PA side chains on constant chain length siloxane polymers on $\mathrm{CO}_{2}$-solubility. We have also examined the phase behavior of the non-functionalized polymer $(z=0)$. The phase behavior data for PDMA-functional siloxane polymers shown in Fig. 8 illustrate that $z=1$ is the optimum degree of substitution, with $z=2$ being very close to the $z=1$ result. The $z=5$ and $z=11$ PDMA-functional copolymers were immiscible up to the pressure of $45 \mathrm{MPa}$ at $295 \mathrm{~K}$, but became miscible with $\mathrm{CO}_{2}$ at a temperature of $311 \mathrm{~K}$ (Fig. 8). This implies that PDMA-functionalized siloxane becomes increasingly less $\mathrm{CO}_{2}$-soluble with increasing degree of substitution. Moreover, these polymers exhibit UCST (upper critical solution temperature) behavior (the miscibility pressure shifts to lower values as the temperature increases). UCST

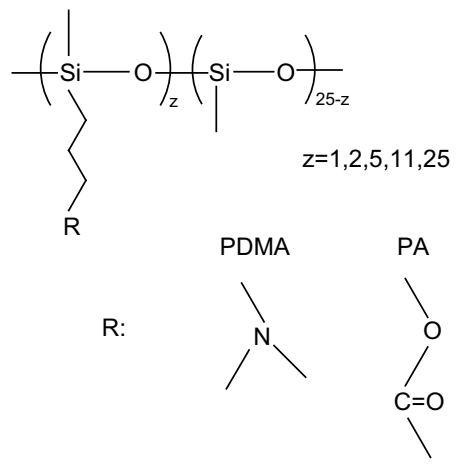

Fig. 7. Structure of propyl dimethylamine (PDMA)- and propyl acetate (PA)-functionalized siloxane copolymers.

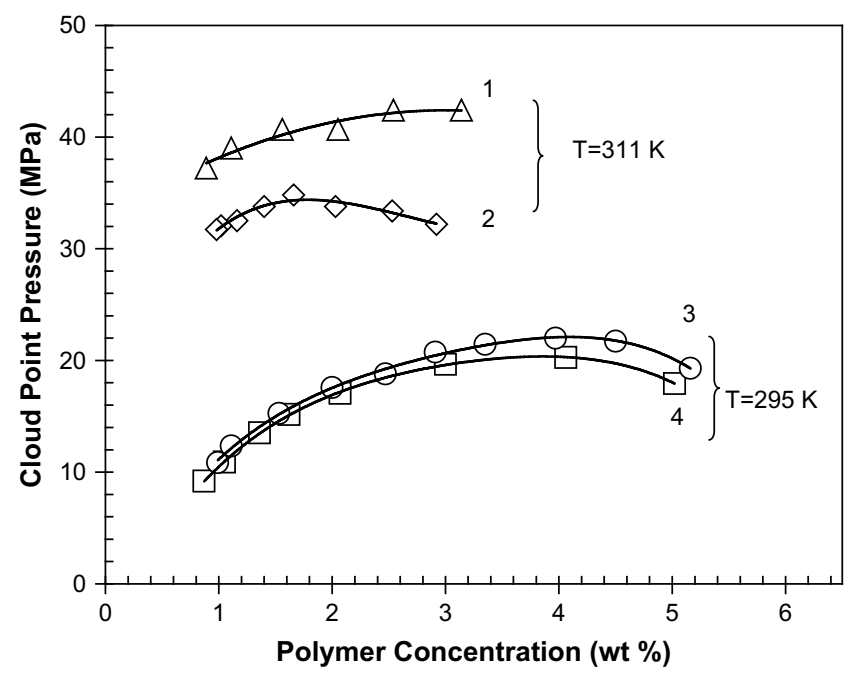

Fig. 8. Phase behavior of propyl dimethylamine (PDMA)-functional siloxane copolymers at different degrees of substitution and temperatures. 1) $z=11, T=311 \mathrm{~K}, 2) z=5$, $T=311 \mathrm{~K}, 3) z=2, T=295 \mathrm{~K}, 4) z=1, T=295 \mathrm{~K}$. The polymers with $z=5$ or 11 did not dissolve in $\mathrm{CO}_{2}$ at $\mathrm{T}=295 \mathrm{~K}$.

behavior in turn implies that the heat of mixing is positive, $\Delta H_{\mathrm{m}}>0$, and that at high temperature the $T \Delta S_{\mathrm{m}}$ term becomes large enough for the Gibbs free energy of mixing to become negative, $\Delta G_{\mathrm{m}}=\Delta H_{\mathrm{m}}-T \Delta S_{\mathrm{m}}$. The positive heat of mixing, arising from strong polymer-polymer interactions, is consistent with our $a b$ initio calculations showing strong self-interactions between the trimethylamine moieties (Table 2). We note that the fully PDMA-functional siloxane polymer $(z=25)$ was immiscible with $\mathrm{CO}_{2}$ at $295 \mathrm{~K}$ and $311 \mathrm{~K}$ at pressures lower than $45 \mathrm{MPa}$.

Our previous experimental work identified the optimum degree of substitution for PA-functionalized siloxane as $z=5$ [6]. We plot the cloud point pressures for the optimum PDMA $(z=1)$ and PA $(z=5)$ substituted siloxanes in Fig. 9. The cloud point curve for the PDMA-functional siloxane polymer lies above that for the PAfunctional siloxane polymer, even though the latter has a larger molecular weight, due to the higher degree of substitution of the

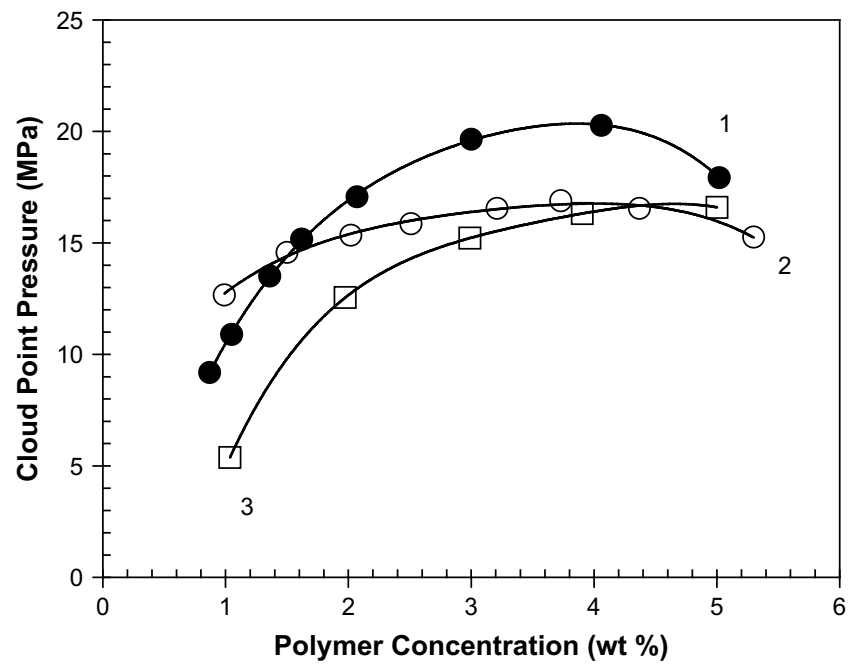

Fig. 9. Comparison of phase behavior of propyl dimethylamine-functional (PDMA) and propyl acetate (PA)-functional siloxane copolymers at their optimum number of substitution with the PDMS base polymer at 295 K. 1) $(z=1)$ Propyl dimethylaminefunctional (PDMA), 2) $(z=5)$ propyl acetate (PA)-functional siloxane copolymers, 3 ) $(z=0)$ PDMS base polymer. 


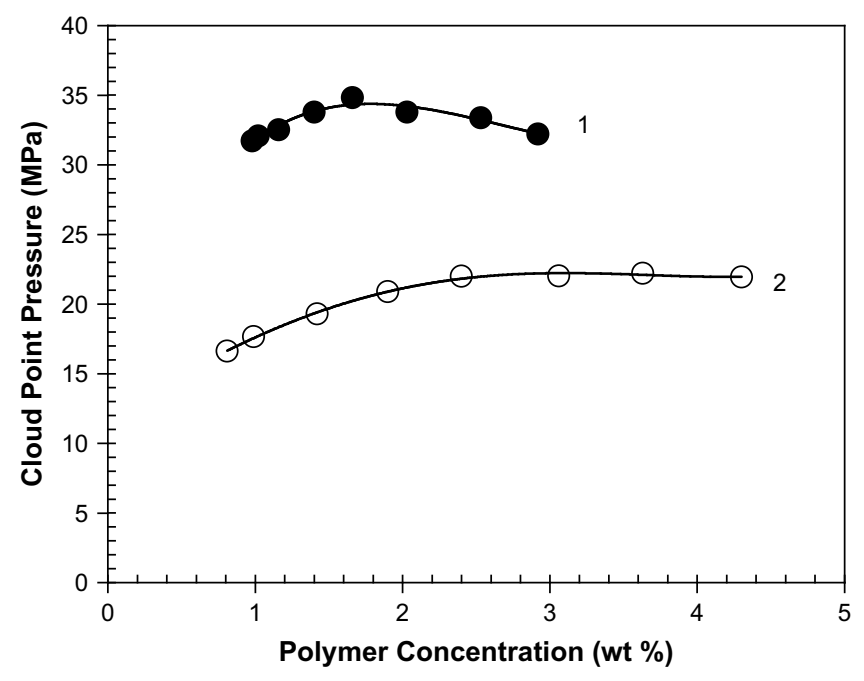

Fig. 10. Comparison of phase behavior of $(z=5)$-functional siloxane copolymers at $311 \mathrm{~K}, 1$ ) propyl dimethylamine (PDMA) 2) propyl acetate (PA).

acetates. Also, the PDMA- and PA-functional polymers are miscible at higher pressures than the PDMS base polymer $(z=0)$, especially at low polymer concentrations. These results lend credence to the hypothesis that the self-interactions between functional groups in the side chains have a larger impact on the miscibility of polymers with $\mathrm{CO}_{2}$ than the $\mathrm{CO}_{2}$-polymer cross interactions. The crossover in miscibility pressures between PDMA and PA substituted siloxanes seen in Fig. 9 at about $1.5 \mathrm{wt} \%$ is due to the increased importance of molecular weight at low concentrations, where polymer selfinteractions are mitigated.

We now compare the change in cloud point pressures as a function of temperature for $(z=5)$ PDMA-functional and the $(z=5)$ PA-functional siloxane copolymers. The cloud point curves for the $(z=5)$ PA-functional siloxane copolymers at $295 \mathrm{~K}$ and $311 \mathrm{~K}$ are plotted in Figs. 9 and 10, respectively. We note that the cloud point curve for the PA-functional PDMS is shifted to higher pressures at $311 \mathrm{~K}$, indicating LCST (lower critical solution temperature) behavior. This implies that $\Delta H_{\mathrm{m}}<0$ and becomes less negative with increasing temperature. The decrease in solubility (increase in cloud point pressure) with increasing temperature is a result of a decrease in the density of $\mathrm{CO}_{2}$ as one goes from a liquid $(295 \mathrm{~K})$ to a supercritical fluid $(311 \mathrm{~K}$, pressure $>7.4 \mathrm{MPa})$.

The cloud point curves at $311 \mathrm{~K}$ for both PDMA- and PA-functionalized polymers, both with $z=5$, are plotted in Fig. 10 . We see that the PA-functionalized siloxane has significantly lower miscibility pressures than PDMA-functionalized siloxane at the same degree of substitution and about the same molecular weight.

These results of the modified PDMS oligomers confirm the findings associated with the insolubility of the aminated hydrocarbon polymers; despite the favorable thermodynamic Lewis acid-Lewis base interactions between $\mathrm{CO}_{2}$ and tertiary amine or pyridine groups, amine-amine self-interactions are responsible for diminished $\mathrm{CO}_{2}$-solubility of amine-containing hydrocarbon polymers relative the oxygenated hydrocarbon polymer PVAc.

\section{Conclusions}

In an attempt to identify a Lewis base group capable of inducing $\mathrm{CO}_{2}$-solubility to a hydrocarbon-based polymer, seven hydrocarbon-based polymers possessing tertiary amine groups either in the backbone or in the pendant chain were designed. All of the polymers were insoluble in $\mathrm{CO}_{2}$ despite the highly " $\mathrm{CO}_{2}$-philic" nature of the tert-amine group. We also carried out complementary quantum mechanical calculations to explain the results at an atomic-level. Although our ab initio calculations suggest that $\mathrm{CO}_{2}-$ amine interactions are even stronger than $\mathrm{CO}_{2}$-carbonyl interactions (known to promote the $\mathrm{CO}_{2}$-solubility of oxygen-rich polymers such as polyvinyl acetate), tert-amine groups do not impart any $\mathrm{CO}_{2}$-solubility to the polymers. The calculations reveal that self-interactions between the amine moieties are energetically more favorable than the $\mathrm{CO}_{2}$-moiety interactions. Hence, dominance of polymer-polymer self-interactions over polymer- $\mathrm{CO}_{2}$ cross interactions is most likely responsible for the tert-amine group not imparting solubility to the hydrocarbon-based polymers. Phase behavior of PDMA-functional siloxane polymers also demonstrated that inclusion of tert-amine moieties in the polymer increases the cohesive energy density of PDMS polymers (even more than does the acetate moiety), resulting in the diminished solubility of tert-aminated PDMS in $\mathrm{CO}_{2}$.

\section{Acknowledgement}

Funding for this work has been provided by the National Science Foundation and the U.S. Department of Energy through the National Energy Technology Laboratory under Grant No. 41817M203841817M2000.

\section{References}

[1] O'Neill ML, Cao Q Fang M, Johnston KP, Wilkinson SP, Smith CD, et al. Solubility of homopolymers and copolymers in carbon dioxide. Ind Eng Chem Res 1998:37:3067.

[2] Enick R, Beckman E, Yazdi A, Krukonis V, Schonemann H, Howell J. Phase behavior of $\mathrm{CO}_{2}$-perfluoropolyether oil mixtures and $\mathrm{CO}_{2}$-perfluoropolyether chelating agent mixtures. J Supercrit Fluids 1998;13:121-6.

[3] Zhao X, Watkins R, Barton SW. Strategies for supercritical $\mathrm{CO}_{2}$ fractionation of polydimethylsiloxane. J Appl Polym Sci 1995;55:773.

[4] Xiong Y, KiranMiscibility E. Density and viscosity of poly(dimethylsiloxane) in supercritical carbon-dioxide. Polymer 1995;36:4817.

[5] Hoefling TA, Newman DA, Enick RM, Beckman EJ. Effect of structure on the cloud-point curves of silicone-based amphiphiles in supercritical carbon dioxide. J Supercrit Fluids 1993;6:165.

[6] Kilic S, Michalik S, Wang Y, Johnson JK, Enick RM, Beckman EJ. Effect of grafted Lewis base groups on the phase behavior of model poly(dimethyl siloxanes) in $\mathrm{CO}_{2}$. Ind Eng Chem Res 2003;42:6415.

[7] Yee GG, Fulton JL, Smith RD. Fourier transform infrared spectroscopy of molecular interactions of heptafluoro-1-butanol or 1-butanol in supercritical carbon dioxide and supercritical ethane. J Phys Chem 1992;96:6172.

[8] Dardin A, DeSimone JM, Samulski ET. Fluorocarbons dissolved in supercritical carbon dioxide. NMR evidence for specific solute-solvent interactions. J Phys Chem B 1998;102:1775.

[9] Bothner-By AA, Glick RE. Specific medium effects in nuclear magnetic resonance spectra of liquids. J Am Chem Soc 1956;78:1071.

[10] Yonker CR, Palmer BJ. Investigation of $\mathrm{CO}_{2} /$ fluorine interactions through the intermolecular effects on the $1 \mathrm{H}$ and $19 \mathrm{~F}$ shielding of $\mathrm{CH}_{3} \mathrm{~F}$ and $\mathrm{CHF}_{3}$ at various temperatures and pressures. J Phys Chem A 2001;105:308.

[11] Cece A, Jureller SH, Kerscher JL, Moschner KF. Molecular modeling approach for contrasting the interaction of ethane and hexafluoroethane with carbon dioxide. J Phys Chem 1996;100:7435.

[12] Diep P, Jordan KD, Johnson JK, Beckman EJ. $\mathrm{CO}_{2}$-fluorocarbon and $\mathrm{CO}_{2}-$ hydrocarbon interactions from first-principles calculations. J Phys Chem A 1998;102:2231

[13] Raveendran P, Wallen SL. Exploring $\mathrm{CO}_{2}$-philicity: effects of stepwise fluorination. J Phys Chem B 2003;107:1473.

[14] Kilic S, Michalik S, Wang Y, Johnson JK, Enick RM, Beckman EJ. Phase behavior of oxygen-containing polymers in $\mathrm{CO}_{2}$. Macromolecules 2007;40(4):1332.

[15] Kirby CF, McHugh MA. Phase behavior of polymers in supercritical fluid solvents. Chem Rev 1999;99:565.

[16] Baradie B, Shoichet MS, Shen Z, McHugh M, Hong L, Wang Y, et al. Synthesis and solubility of linear poly(tetrafluoroethylene-co-vinyl acetate) in dense $\mathrm{CO}_{2}$ : experimental and molecular modeling results. Macromolecules 2004;37:7799-807.

[17] Kazarian SG, Vincent MF, Bright FV, Lioota CL, Eckert CA. Specific intermolecular interaction of carbon dioxide with polymers. J Am Chem Soc 1996;118:1729.

[18] Dobrowolski JC, Jamroz MH. Infrared evidence for carbon dioxide electron donor-acceptor complexes. J Mol Struct 1992;275:211. 
[19] Reilly JT, Bokis JP, Donohue MD. An experimental investigation of Lewis acidbase interactions of liquid carbon dioxide using Fourier-Transform Infrared (FT-IR) spectroscopy. Int J Thermophys 1995;16:599.

[20] Nelson MR, Borkman RF. Ab initio calculations on $\mathrm{CO}_{2}$ binding to carbonyl groups. J Phys Chem 1998;102:7860.

[21] Sarbu T, Styranec TJ, Beckman EJ. Design and synthesis of low cost, sustainable $\mathrm{CO}_{2}$-philes. Ind Eng Chem Res 2000;39:4678.

[22] Sarbu T, Styranec TJ, Beckman EJ. Non-fluorous polymers with very high solubility in supercritical $\mathrm{CO}_{2}$ down to low pressures. Nature 2000;405:165.

[23] Hu Y, Chen W, Xu L, Xiao J. Carbonylated phosphines as ligands for catalysis in supercritical $\mathrm{CO}_{2}$. Organometallics 2001;20:3206.

[24] Raveendran P, Wallen SL. Sugar acetates as novel, renewable $\mathrm{CO}_{2}$-philes. J Am Chem Soc 2002;124:7274.

[25] Potluri VK, Xu J, Enick R, Beckman EJ, Hamilton AD. Peracetylated sugar derivatives show high solubility in liquid and supercritical carbon dioxide. Org Lett 2002;4:2333.

[26] Shen Z, McHugh MA, Xu J, Belardi J, Kilic S, Mesiano A, et al. $\mathrm{CO}_{2}$-solubility of oligomers and polymers that contain the carbonyl group. Polymer 2003;44:1491.

[27] Raveendran P, Wallen SL. Cooperative $\mathrm{C}-\mathrm{H} \cdots \mathrm{O}$ hydrogen bonding in $\mathrm{CO}_{2}-$ Lewis base complexes: implications for solvation in supercritical $\mathrm{CO}_{2}$. J Am Chem Soc 2002;124:12590.

[28] Van Ginderen P, Herrebout WA, van der Veken BJ. van der Waals complex of dimethyl ether with carbon dioxide. J Phys Chem A 2003;107(28):5391.

[29] Danten Y, Tassaing T, Besnard M. Vibrational spectra of $\mathrm{CO}_{2}$-electron donoracceptor complexes from ab initio. J Phys Chem A 2002;106(48):11831.

[30] Wang Y, Hong L, Kim I, Paik I-H, Crosthwaite JM, Hamilton AD, et al. Design and evaluation of non-fluorous $\mathrm{CO}_{2}$-soluble polymers, in preparation.

[31] Tapriyal D, Johnson K, Enick R. Poly(vinyl acetate), poly(1-O-(vinyloxy) ethyl$2,3,4,6$-tetra-O-acetyl- $\beta$-D-glucopyranoside) and amorphous poly(lactic acid) are the most $\mathrm{CO}_{2}$-soluble oxygenated hydrocarbon-based polymers. J Supercrit Fluids 2008;46:252-7.

[32] Jamroz MH, Dobrowolski JC, Bajdor K, Borowiak MA. Ab-initio study of the $\mathrm{Nu}\left(\mathrm{CO}_{2}\right)$ mode in EDA complexes. J Mol Struct 1995;349:9.

[33] Park Y. Specific interactions between phosphorus compounds and carbon dioxide: $a b$ initio approach. J Supercrit Fluids 2005;36:154.

[34] Kilic S. Engineering of polymers to thicken carbon dioxide: a systematic approach. PhD Dissertation, University of Pittsburgh, 2003.
[35] Meredith JC, Johnston KP, Seminario Jorge M, Kazarian Sergei G, Eckert Charles A. Quantitative equilibrium constants between $\mathrm{CO}_{2}$ and Lewis bases from FTIR spectroscopy. J Phys Chem 1996;100:10837.

[36] Milet A, Korona T, Moszynski R, Kochanski E. Anisotropic intermolecular interactions in van der Waals and hydrogen-bonded complexes: what can we get from density functional calculations? J Chem Phys 1999;111:7727-35.

[37] Gutmann V. Solvent effects on the reactivities of organometallic compounds. Coord Chem Rev 1976;18:225.

[38] Kachi Y, Tsukahara T, Kayaki Y, Ikariya T, Sato J, Ikeda Y. Raman spectral shifts of $\mathrm{CO}_{2}$ as measure of $\mathrm{CO}_{2}$-philicity of solutes in supercritical carbon dioxide. J Supercrit Fluids 2007;40(1):20.

[39] Linert W, Fukuda Y, Camard A. Chromotropism of coordination compounds and its applications in solution. Coord Chem Rev 2001;218:113.

[40] Vaidya PD, Kenig EY. Acceleration of $\mathrm{CO}_{2}$ reaction with $\mathrm{N}, \mathrm{N}-$ diethylethanolamine in aqueous solutions by piperazine. Ind Eng Chem Res 2008;47:34.

[41] Brandup J, Immergut EH, Grulke EA. Polymer handbook. 4th ed. New York: Wiley; 1999.

[42] Li D, Brittain WJ. Synthesis of poly(N,N-dimethylacrylamide) via nitroxidemediated radical polymerization. Macromolecules 1998;31:3852.

[43] Smith MB, March J. March's advanced organic chemistry: reactions, mechanisms, and structure. Wiley, John \& Sons; 2000.

[44] Kwiatkowski S, Jeganathan A, Tobin T, Watt DS. A synthesis of N-substituted $\beta$ alanines: Michael addition of amines to trimethylsilyl acrylate. Synthesis 1989;12:946.

[45] Farahani M, Antonucci JM, Karam LR. A GC-MS study of the addition reaction of arylamines with acrylic monomers. J Appl Polym Sci 1998;67:1545.

[46] Frisch MJ, Trucks GW, Schlegel HB, Scuseria GE, Robb MA, Cheeseman JR, et al. Gaussian 03, revision C.02. Wallingford, CT: Gaussian, Inc.; 2004.

[47] Boys SF, Bernardi F. The calculation of small molecular interactions by the differences of separate total energies. Some procedures with reduced errors. Mol Phys 1970;19:553-66.

[48] Feller D, Jordan KD. Estimating the strength of the water/single-layer graphite interaction. J Phys Chem A 2000;104:9971-5.

[49] Becke AD. Density-functional thermochemistry. III. The role of exact exchange. J Chem Phys 1993;98:5648-52.

[50] Sauer BB, Dee GT. Surface tension and melt cohesive energy density of polymer melts including high melting and high glass transition polymers. Macromolecules 2002;35:7024. 